\title{
Evaluation of Interactive Computerized Training to Teach Paraprofessionals to Implement Errorless Discrete Trial Instruction
}

\author{
Kristina R. Gerencser ${ }^{1,2}$ (D) Thomas S. Higbee ${ }^{1} \cdot$ Bethany P. Contreras $^{1,3}$. \\ Azure J. Pellegrino ${ }^{4}$. Summer L. Gunn ${ }^{1}$
}

(c) Springer Science+Business Media, LLC, part of Springer Nature 2018

\begin{abstract}
Efficient and effective training strategies for paraprofessionals in special education settings face many challenges. Interactive computerized training (ICT) - a selfpaced program that incorporates audio narration, video models, interactive activities, and competency checks - is one potential solution. ICT has been successful in training college students and special education teachers to implement discrete trial instruction (DTI), but its effectiveness to train paraprofessionals is unknown. Using a multiple-baseline design, we evaluated the feasibility of ICT, to train six paraprofessionals to implement DTI with an errorless learning procedure. Following ICT, the fidelity of implementation of DTI increased for all participants when implemented with a student in their classroom; however, competency varied. We added additional training components that progressed from low to more intensive feedback delivered remotely in attempt to increase fidelity to $90 \%$ or higher implementation. We also evaluated generalization to novel instructional programs and maintenance of instruction in the absence of feedback.
\end{abstract}

Keywords Computer training · Discrete trial instruction · Interactive computerized training $\cdot$ Paraprofessionals $\cdot$ Staff training

Kristina R. Gerencser

kristina.gerencser@choa.org

1 Department of Special Education and Rehabilitation, Utah State University, Logan, UT, USA

2 Marcus Autism Center, Emory University, 1920 Briarcliff Road NE, Atlanta, GA 30329, USA

3 Middle Tennessee State University, Murfreesboro, TN, USA

4 University of Kansas, Lawrence, KS, USA 


\section{Introduction}

In the USA, over 400,000 paraprofessionals provide educational services to individuals with disabilities between 3 and 21 years of age (U.S. Department of Education 2014). Thus, paraprofessionals play an essential role in teaching individuals diagnosed with autism spectrum disorder (ASD) and other developmental disabilities. Although special education teachers are generally responsible for designing students' educational goals, properly trained paraprofessionals can assist the teacher in a variety of ways, such as implementing interventions, teaching, and monitoring progress (Boomer 1994). Paraprofessionals frequently have lower levels of education compared to teachers and are rarely provided with the specialized training necessary to implement evidence-based interventions to support students with disabilities (Riggs and Mueller 2001). Without adequate training, paraprofessionals can unintentionally create prompt dependency, limit academic growth, and reinforce problem behavior. With the growing reliance on paraprofessionals as instructors, it is imperative that paraprofessionals be well trained to deliver high-quality instruction.

One common instructional strategy that paraprofessionals might be responsible for implementing is discrete trial instruction (DTI). Each learning opportunity, or discrete trial, involves the systematic presentation of instructional components (gaining the student's attention, presenting an instruction, prompting accurate responding, delivering a consequence, etc.). Although there is a great need for well-trained paraprofessionals to implement behavior analytic interventions such as DTI, there remains a discrepancy in the implementation of such evidencebased procedures. According to research conducted by Joyce and Showers (2002) and Fixsen et al. (2005), as a field we have identified many evidence-based strategies; however, we continue to fall behind in the implementation of these interventions in desired settings, such as public schools, with high procedural integrity.

In order to achieve high levels of integrity to implement DTI, intensive staff training is generally required. Often staff training is comprised of face-to-face training methods, such as behavior skills training (BST, i.e., instructions, modeling, rehearsal, and feedback) delivered from a qualified professional (Sarokoff and Sturmey 2004). However, this may not be practical or achievable for school districts to provide training to paraprofessionals on evidence-based interventions. A potential alternative method, which has had promising results in teaching undergraduate students (Pollard et al. 2014) and special education teachers (Higbee et al. 2016) to implement DTI, is through interactive computerized training (ICT). Interactive computerized training incorporates components of BST into a self-paced training package that is accessible on a computer or an Internet site. This format does not require a professional and trainee to be present for instruction to occur. Content is divided into modules that include narrated slides with written text, graphics, and video examples of the target skills. In addition, competency checks and interactive activities (e.g., self-guided practice opportunities) are embedded to provide the trainee with an opportunity to rehearse the targeted skills and receive feedback on the content. Following the completion of the 
training, trainees are instructed to demonstrate the skill with a confederate (i.e., an adult who plays the role of a client) or with a client.

Pollard et al. (2014) investigated the effectiveness of using ICT to teach four undergraduate students to implement DTI. The ICT content was divided into four modules and hosted on an online course management site. In addition to the general components of DTI, participants were taught to use a least-to-most prompting procedure. Following each module, participants role-played with a confederate for 20 trials interspersed across three instructional programs (imitation, receptive shape identification, and expressive color identification). The role-play sessions were designed such that the confederate engaged in correct and error responses so that the participant had the opportunity to implement each component of DTI trained in the modules. For example, each role-play session included two occasions where the confederate responded incorrectly for two consecutive trials to assess the participants' ability to increase the prompts within the least-to-most prompting procedure (e.g., move from a gesture prompt to a physical prompt). Following the ICT, participants demonstrated significant increases in DTI implementation and all participants reached the mastery criterion (i.e., $85 \%$ or higher across two consecutive sessions). For all participants, DTI skills generalized to a child with ASD and to untrained, but similar, instructional programs. One participant, however, required a brief live feedback session in order to meet the mastery criterion with a student with ASD.

Higbee et al. (2016) replicated and extended Pollard et al. (2014) in a two-part international study with four undergraduate students and four special education teachers. The ICT training modules were the same as Pollard et al., but translated into Brazilian Portuguese. Participants' fidelity of DTI implementation was measured during role-play sessions with a confederate (undergraduate participants) and during sessions with a student with ASD (all sessions for teachers and generalization probes for undergraduate participants). Following the completion of the ICT training, all participants' fidelity substantially increased. Five out of the eight participants required feedback on data collection or prompting to reach criterion. All feedback was given in person. Participants' responding generalized to untrained instructional programs and maintenance of the skills remained at criterion (i.e., $85 \%$ or higher) for three of the four teachers at a one-month follow-up.

The current literature demonstrates the potential utility of ICT as an alternative solution to the barriers that are often associated with more traditional training methods (i.e., those mediated by a professional). However, the effect of this training method to teach paraprofessionals to implement DTI is unknown. In addition, the previous studies only assessed a simplified version of the common components that typically occur during a teaching session. These studies did not have participants conduct preference assessments to identify highly preferred items for correct responses and did not teach participants to fade their prompts to promote independent responding following error correction. Furthermore, these studies involved the delivery of in person feedback for participants who required additional training in order to meet the performance criterion. DTI is a complex teaching procedure that may require more feedback and coaching for some individuals to master. However, providing face-to-face feedback and coaching limits one of the main purported advantages of ICT-eliminating the need for a professional to be physically 
present. It is possible that performance feedback could be effective if it was delivered remotely using a video conferencing system rather than in person.

Given these limitations and the various barriers (e.g., time, money, resources) school districts face in training, further investigation of ICT to train paraprofessionals is warranted. Therefore, the purpose of this study was threefold: (a) to extend the existing literature on ICT to investigate the utility of the technology to teach paraprofessionals to implement DTI with students diagnosed with developmental disabilities, (b) to teach the paraprofessionals to use a flexible error correction and prompting procedure to promote independence, and (c) to evaluate the use of feedback and coaching delivered remotely for paraprofessionals whom failed to meet the performance criterion with ICT alone.

\section{Method}

\section{Participants}

Three special education preschool classrooms from a rural school district participated in this study. To be included, each classroom needed to meet the following inclusion criteria: (a) a teacher willing to help conduct research sessions and commit to the weekly session requirements, (b) two paraprofessionals willing to participate, and (c) two students who met the inclusion criteria (see student criteria below). Paraprofessionals were eligible to participate if they had no formal training in DTI and if their fidelity of implementation was below the mastery criterion of $90 \%$ during baseline. Two paraprofessionals from each of the three classrooms met the inclusion criteria for this study, for a total of six paraprofessionals. Paraprofessionals where all Caucasian females that ranged from 38 to 70 years $(\mathrm{M}=51.3$ years $)$ and worked $25 \mathrm{~h}$ per week earning between $\$ 13$ and $\$ 17$ per hr (Table 1). Three participants

Table 1 Participant demographics

\begin{tabular}{|c|c|c|c|c|c|c|c|}
\hline Participant & Gender & Age & Ethnicity & Education & $\begin{array}{l}\text { Years } \\
\text { employed }\end{array}$ & $\begin{array}{l}\text { Years in } \\
\text { current } \\
\text { class }\end{array}$ & Student diagnosis \\
\hline Danielle & $\mathrm{F}$ & 46 & Caucasian & $\begin{array}{l}\text { High school } \\
\text { diploma }\end{array}$ & 9 & 9 & Down syndrome \\
\hline Jody & $\mathrm{F}$ & 70 & Caucasian & Some college & 15 & 10 & $\begin{array}{c}\text { Developmental } \\
\text { disability }\end{array}$ \\
\hline Candy & $\mathrm{F}$ & 42 & Caucasian & Bachelor's degree & 7 & 1 & Down syndrome \\
\hline Poppi & $\mathrm{F}$ & 57 & Caucasian & $\begin{array}{l}\text { High school } \\
\text { diploma }\end{array}$ & 10 & 8 & $\begin{array}{l}\text { Developmental } \\
\text { disability }\end{array}$ \\
\hline Nancy & $\mathrm{F}$ & 55 & Caucasian & $\begin{array}{l}\text { High school } \\
\text { diploma }\end{array}$ & 20 & 5 & $\begin{array}{l}\text { Developmental } \\
\text { disability }\end{array}$ \\
\hline Vanessa & $\mathrm{F}$ & 38 & Caucasian & Associate degree & 8 & 4 & $\begin{array}{l}\text { Autism spectrum } \\
\text { disorder }\end{array}$ \\
\hline
\end{tabular}

$F$ female 
had some college education, and all participants had extensive work experience as a paraprofessional $(\mathrm{M}=11.5$ years, range 7-20 years). Each paraprofessional (hereafter referred to as participant) was paired with a student with a developmental disability to form two participant-student dyads per classroom. Students were included in this study if they demonstrated the following skills: (a) independently sitting in their chair for 5 min during instructional activities; (b) minimal challenging behavior observed during instructional activities; and (c) echoing, labeling, or requesting with one- to two-word phrases.

\section{Setting and Materials}

\section{Teaching Environment}

All sessions were conducted in either a small room adjacent to the participants' classroom, or in an individualized work space in the corner of the classroom with cubicle walls to minimize distractions and prevent participants from observing each other. The instructional area included a small table, two chairs, and a bin of teaching materials. A research assistant used a video camera to record all sessions.

\section{Teaching Materials}

During each session, the participant was given a bin of materials required to implement DTI. The bin contained materials specific to the student they were paired with, including: (a) five preferred edibles and five preferred tangible items (identified by the classroom teacher), (b) flashcards, and (c) a curriculum binder. The flashcards varied by dyad, but generally included pictures of common objects that would be used to teach receptive and expressive labeling. The curriculum binder included preference assessment data sheets, instructional program sheets for three different programs, and corresponding data sheets for the instructional programs. The instructional program sheets included written information necessary for teaching the specified skill, such as the vocal instruction to be used to teach the target, a brief description of how to teach the program, the materials to be used, the prompt sequence, the fading and error correction rules, the data collection rules, and a list of teaching targets to be taught. The corresponding data sheet included the vocal instruction and an operational definition of the correct response for each target.

Six instructional programs were used to assess participant's implementation of DTI. Three programs were used for post-ICT sessions and included: nonverbal imitation (i.e., "Do this (model action)."), receptive identification (e.g., "Touch cat."), and expressive identification (i.e., "What is it?"). Three were used for generalization sessions and included: receptive actions (e.g., "Wave."), match-to-sample (i.e., "Match."), and answering simple questions (e.g., "What is your name?"). Each program contained two teaching targets, and receptive and match-to-sample program targets were presented in an array of three comparison stimuli. A new target was introduced if a target was mastered (i.e., 80\% or higher across four consecutive 
sessions) to ensure that we could evaluate participant's fidelity in using the errorless learning procedure.

\section{Interactive Computerized Training}

Six ICT modules were developed using Adobe Captivate ${ }^{\circledR}$ version 9 software and were accessible on Instructure Canvas (an online course management system). The training modules included audio narration, supported texts and graphics, video models, competency questions, and interactive activities. The content of the modules was developed and modified from preexisting training modules (Pollard et al. 2014) and from a researched self-instructional manual (Severtson and Carr 2012). The modules were designed to provide background information and instruction on how to teach skills using DTI, and each module taught different components of DTI. The specific DTI components that were taught and assessed in this study are listed in Table 2, along with operational definitions for each. Table 3 lists the DTI components there were taught in each module.

Table 3 also lists the additional materials (from a downloadable packet) associated with each module, the number of video models and skills modeled per module, the number of competency checks, and number and type of interactive activities per module. Additional materials included a list of praise statements, program sheets, data sheets, and flash cards to be used during self-guided practice. These materials were the same as those included in the curriculum binder provided throughout the study (described above). Video models were embedded within the modules to demonstrate proper implementation of the DTI components. All video models were created specifically for this ICT, and all involved an adult instructor demonstrating the relevant skill with a child with ASD.

Each module included competency checks, which assessed the participants' knowledge throughout the module. Competency checks were short questions based on the content from each module and were either multiple choice or fill-in-the-blank questions (a complete list of the competency check questions is available upon request from the first author). Each module also included interactive activities and self-guided activities to allow the participant opportunities to practice components of DTI. These activities were more complex than competency checks and included drag-and-drop activities, manipulation of stimuli, and practice collection of session data. Following each competency check question and interactive/self-guided activity, the ICT provided the participant with brief feedback regarding their performance. If the participant completed the activity correctly, they were shown a screen with positive feedback that also summarized why their answer was correct. If the participant completed an activity incorrectly, they were shown a screen with corrective feedback, and then the ICT returned the participant to the content slides. The participant was then required to review those slides, and the competency check or interactive activity was repeated. Participants were required to complete each activity correctly in order to advance to the next content slide and next module.

The ICT modules provided information on how to implement DTI for teaching imitation, receptive identification, and expressive identification. The ICT did not provide any information or examples of how to use DTI to teach receptive actions, 
Table 2 DTI components and definitions

Target behaviors Definitions

Assessing student preference correctly

(a) Conduct a brief MSWO for edible and tangible reinforcers (as described in Carr et al. 2000) and identify the first and second ranked items (one per session)

Present materials correctly

Receptive identification/match-to-sample programs

(a) 3 flashcards presented in a different order than the previous trial

(b) 3 flashcards were evenly spaced and facing the student

Expressive identification program

Secure student's attention

(a) A single flashcard held up in front of the student

(a) Used a visual shield (hands held up to sides of student's face to guide student's gaze to the participant)

(b) Used the student's name once

(c) Student already attending to materials or instructor prior to instruction

Delivered correct instruction $\left(\mathrm{S}^{\mathrm{D}}\right)$

(a) Used instruction specified on the program/data sheet—no added or omitted words

(b) Spoken in a neutral tone of voice

Waited $5 \mathrm{~s}$ for a response

(a) For independent opportunities, allowed the student $5 \mathrm{~s}$ to respond to the instruction. Did not deliver a prompt, remove materials, or deliver another instruction within those $5 \mathrm{~s}$

(b) For prompted trials, delivered a prompt simultaneously with the instruction and allowed the student $5 \mathrm{~s}$ to respond to the instruction plus prompt. Did not deliver another prompt, remove materials, or deliver another instruction for $5 \mathrm{~s}$

Provided prompt immediately

(a) Present prompt simultaneously with (receptive identification, receptive actions, and match-tosample) or immediately ( $\geq 3 \mathrm{~s}$ ) after (expressive identification, imitation, answering questions) the instruction

Provided the correct prompt level

(a) Probe trials: used least-to-most prompting

(b) Teaching trials: started each target at the prompt level identified from probe trials

(c) Teaching trials: used most-to-least prompting and faded the prompt following two correct responses at the specified prompt level or continued presenting independent opportunities following a correct response

(d) Teaching trials: increased the prompt level following an incorrect response or stayed at the most intrusive prompt level until the student responded correctly 
Table 2 (continued)

\begin{tabular}{|c|c|}
\hline Target behaviors & Definitions \\
\hline Immediately delivers an appropriate consequence & $\begin{array}{l}\text { (a) Correct response: delivered varied praise (dif- } \\
\text { fered from previous statement) and a reinforcer } \\
\text { (first or second ranked item from MSWO or item } \\
\text { requested by the student) within } 5 \mathrm{~s} \\
\text { (b) Incorrect response: delivered feedback within } 5 \mathrm{~s} \\
\text { by saying "try again," breaking eye contact, or a } \\
\text { combination }\end{array}$ \\
\hline Removes materials & (a) Removes materials prior to starting a new trial \\
\hline Correctly record data & $\begin{array}{l}\text { (a) Probe trials: circle the correct prompt level to be } \\
\text { used for teaching } \\
\text { (b) Teaching trials: after every trial, records the } \\
\text { correct prompt level and student response (e.g., } \\
\text { I+, P-) }\end{array}$ \\
\hline Inter-trial interval & $\begin{array}{l}\text { (a) Presents another instruction within } 5 \mathrm{~s} \text { from } \\
\text { the last delivered consequence ( } 5 \mathrm{~s} \text { following an } \\
\text { edible reinforcer, } 5 \mathrm{~s} \text { following the return of a } \\
\text { tangible reinforcer, or } 5 \mathrm{~s} \text { following an informal } \\
\text { preference assessment) }\end{array}$ \\
\hline Correct interspersal (teaching trials only) & $\begin{array}{l}\text { (a) Following a correct response, moved to another } \\
\text { target from within the program or across another } \\
\text { program } \\
\text { (b) Following an incorrect response, stayed with tar- } \\
\text { get until student responds correctly or conducted a } \\
\text { maximum of } 10 \text { trials }\end{array}$ \\
\hline $\begin{array}{l}\text { Correctly ends teaching for each target (teaching } \\
\text { trials only) }\end{array}$ & $\begin{array}{l}\text { (a) Conducted a minimum of } 5 \text { trials ending teach- } \\
\text { ing either at the starting prompt level identified } \\
\text { from probe trial or less or at a maximum of } 10 \\
\text { trials }\end{array}$ \\
\hline
\end{tabular}

matching, or answering simple questions. The latter three types of instructional programs were reserved to test for generalization of implementation of DTI. That is, the ICT directly taught the participant to use DTI to teach the three types of skills which were assessed during their teaching sessions and did not teach the participant to use DTI to teach the skills assessed during generalization sessions.

Completion and duration of the ICT was tracked by having each participant record their start and stop times using the interactive quiz feature of Instructure Canvas. The participants completed the modules on a computer with Internet access either at their school or at home (or both). No one else was present while they completed the modules; therefore, we were unable to collect additional data on their level of engagement with the modules (other than the total duration to complete the modules). Following the completion of the study, each participant received compensation of $\$ 50$ from the school district. The ICT modules taught the participant to conduct teaching sessions in which they would complete the following sequence of behaviors: (a) conduct two brief multiple-stimulus without replacement (MSWO) preference assessments to identify the top two preferred edible and tangible reinforcers (Carr et al. 2000), (b) conduct probe trials to 


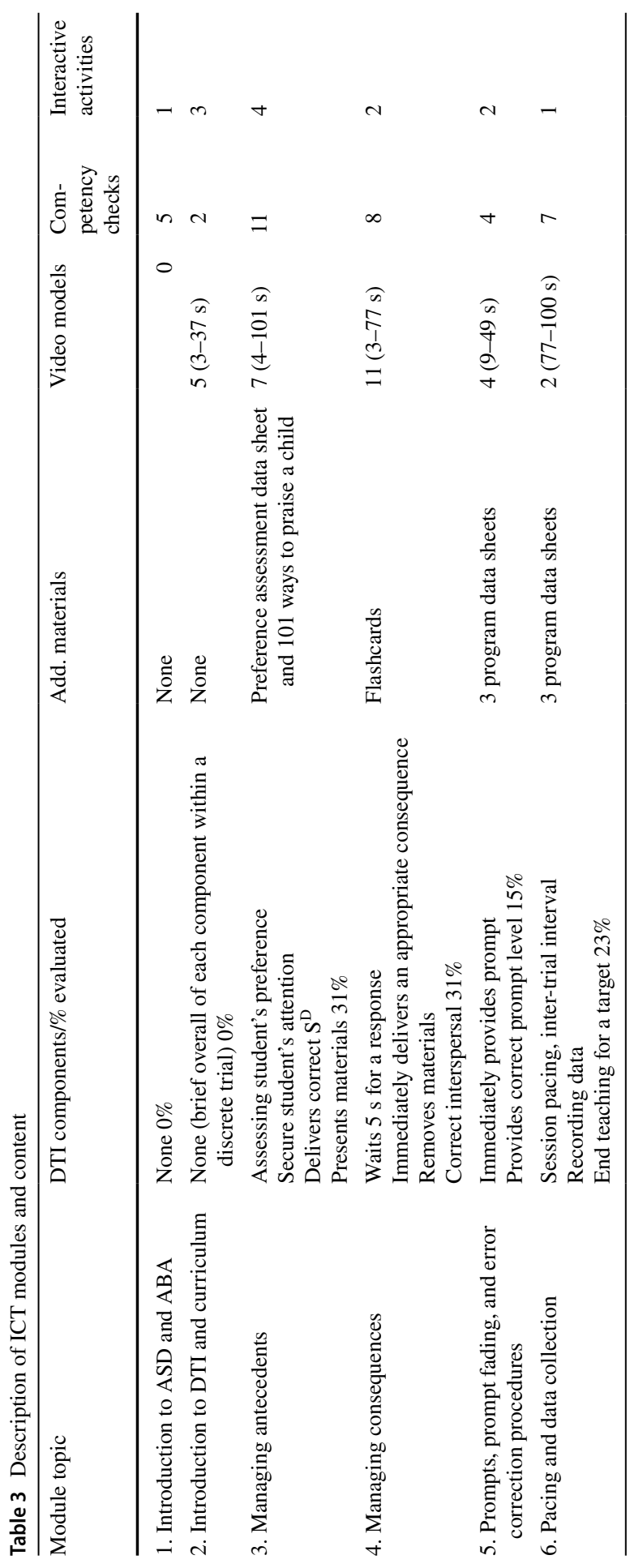


determine the starting prompt level for each target, and (c) conduct teaching trials interspersing targets from within and across the three instructional programs. Each of these general components is outlined below.

Conduct Preference Assessments The participants were taught to conduct brief MSWO preference assessments based on the procedures outlined in Carr et al. (2000). Participants conducted one assessment for edible items and one for tangible items, and each array consisted of five items.

Conduct Probe Trials The participants were taught to conduct probe trials for each target to identify the appropriate prompt level to use when teaching each target. The procedures for conducting the probe trials were based on those presented in (modified from Severtson and Carr 2012). Probe trials were to be conducted using least-to-most prompting in order to identify the level of prompting at which the student was most likely to be successful. Participants were taught to conduct each probe trial using the following DTI components: (a) secure student's attention, (b) provide the instruction and materials (if necessary), (c) wait $5 \mathrm{~s}$ for the student to respond (i.e., test for independence), (d) provide an appropriate consequence, and (e) record data for the trial. A correct response was defined as the student responding correctly to the instruction independently or with a prompt within $5 \mathrm{~s}$ of the instruction/prompt being presented. If the student responded correctly, the module taught participants to deliver reinforcement and record an independent response on the data sheet.

An incorrect response was defined as the student responding incorrectly to the instruction (with or without a prompt), or not responding to the instruction within $5 \mathrm{~s}$. If the student responded incorrectly, the module instructed participants to deliver feedback by breaking eye contact and/or saying, "Try again" and recorded an error on the data sheet. Then, another trial was to be presented for that same target with a prompt using a least-to-most prompt hierarchy. For example, for a receptive identification program, the module taught participants to represent the instruction while simultaneously pointing to the correct answer (i.e., partial prompt). If the student responded correctly, participants were instructed to deliver reinforcement and record the prompt level (e.g., "P" for partial prompt). If the student responded incorrectly again, the participants were taught to give feedback (e.g., break eye contact) and record that the student would require a full prompt (i.e., physical prompt) for teaching trials. The module instructed participants not to assess the student's response to a full prompt during probe trials because it was apparent that the student would require a full prompt following an incorrect response to the partial prompt. The module taught participants to continue this process for each of the six teaching targets. Therefore, one to two probe trials would be required per target, for a total of six to 12 total probe trials per session.

Conduct Teaching Trials The participants were taught to conduct teaching trials once they had completed the probe trials for all six targets. The training modules instructed participants to use an errorless learning procedure during teaching 
(modified from Severtson and Carr 2012), to prevent student errors and promote independent responding. The general DTI components were similar to the probe trials, to: (a) secure student's attention, (b) provide the instruction and materials (if necessary), (c) provide a prompt (if necessary), (d) wait $5 \mathrm{~s}$ for the student to respond, (e) provide an appropriate consequence, (f) record data for the trial, (g) and intersperse trials across targets and instructional programs. The type of prompt used varied slightly from program-to-program (e.g., using a full physical prompt to clap hands for an imitation target compared to a full vocal model, "dog," for expressive identification target), but the hierarchy of most-to-least prompts were kept consistent to three levels: full prompt, partial prompt, no prompt (i.e., independent opportunity). The modules provided two rules regarding when to fade prompts and how to correct errors: (a) following two consecutive correct responses at a specified prompt level, fade prompt to the next less intrusive level (e.g., following two correct responses with a full prompt, use a partial prompt on the next trial); and (b) following one incorrect response or no response, increase to the next more intrusive prompt level (e.g., following an incorrect response with a partial prompt, use a full prompt on the next trial).

To help follow the rules, the module instructed participants to score each trial based on the prompt level provided (i.e., $\mathrm{F}=$ full prompt, $\mathrm{P}=$ partial prompt, $\mathrm{I}=$ independent) and based on the student's response (i.e., $+=$ correct response, $-=$ incorrect response). For example, if the student responded correctly to the instruction with a full prompt, the data would be scored as F+. If the student responded incorrectly to the instruction with a partial prompt, the data would be scored $\mathrm{P}-$. Following a correct response, participants were taught to deliver reinforcement (i.e., varied praise paired with an edible/toy ranked first or second from the brief MSWO) and collect data. Next, if a student made a correct response, participants learned to intersperse targets within the same instructional program and/or across the other instructional programs. For example, the participant could conduct a target from the imitation program and then move to a target from the receptive identification program, then conduct another target from the receptive identification program, then go back to a target from the imitation program, and then run a target from the expressive identification program.

If a student responded incorrectly, the module taught participants to provide feedback by breaking eye contact with the student and/or saying, "Try again" while clearing materials (if necessary) and recording the student's response on the data sheet. The participants re-presented that target (with the appropriate prompt) until the student responded correctly or until a maximum of 10 trials were conducted. Following each error, the participant increased the prompt level (i.e., partial prompt, full prompt). When the student responded correctly, the participant was taught to deliver reinforcement and move to another target.

A target was considered completed for that teaching session when a minimum of five trials were completed with the student responding correctly on the last trial at the starting prompt level (identified during the probe trial) or at a lesser prompt level. If at the fifth trial the student made an incorrect response, teaching trials continued, using the prompt and prompt fading procedure rules, until the student responded with a correct response at the starting prompt level or lesser prompt level 
or until a maximum of 10 trials were conducted. A cutoff of 10 teaching trials was used to limit the variability of the number of trials conducted across participants and sessions. Permitting a range of trials to occur allowed us to capture the participants' integrity of implementation of the errorless learning and error correction procedures. In Classroom 1, Danielle conducted an average of 71 trials (range 65-72) in baseline and an average of 59 trials (range 41-72) in treatment and Jody conducted an average of 18 trials (range 10-35) and an average of 41 trials (range 22-48) during treatment. In Classroom 2, Candy conducted an average of 32 trials (range 25-40) during baseline and an average of 48 trials (range 39-58) during treatment and Poppi conducted an average of 38 trials (range 23-45) during baseline and an average of 43 trials (range 34-56) during treatment. Lastly, in Classroom 3, Nancy conducted an average of 50 trials (range 0-63) during baseline and an average of 55 trials (range 36-68) during treatment and Vanessa conducted an average of 37 trials (range 31-51) during baseline and an average of 45 trials (range 36-56) during treatment.

\section{Dependent Measures and Data Collection}

The primary dependent measure for this study was the percentage of DTI components implemented correctly during each teaching session. We measured this using a fidelity checklist which was modified from Pollard et al. (2014) and Fazzio and Martin (2011). The checklist measured each of the components listed in Table 2. Each component of DTI was scored as correctly implemented, incorrectly implemented, or not applicable. Components were scored as correct if the participant implemented them as described in Table 2. If the participants' behavior deviated from the operational definition, or if they did not implement a component at all, it was scored as incorrect. If the participant did not have the opportunity to implement a component (e.g., did not have the opportunity to implement error correction because the student responded correctly), the component was scored as not applicable. The percentage of correct implementation for the session was calculated by dividing the number of correct components by the number of total components (not including those marked as not applicable) and multiplying by 100 .

The number of times a DTI component was scored varied across components and sessions. "Assessing student preference correctly" was assessed two times per session, once for the edible assessment and once for the tangible assessment. All other DTI components were assessed multiple times throughout each session (once per trial). Two types of trials were implemented within each session, probe trials and teaching trials (as described above). "Correct interspersal" was only assessed on teaching trials, and "correctly ends teaching for each target" was assessed once for each target (total of six opportunities per session). All other components were assessed for each trial (both probe and teaching). The total number of trials varied across sessions, depending on the students' responding. There could be between six and 12 probe trials per session and between 30 and 60 teaching trials per sessions (as described above). Because the number of trials could vary across sessions, the denominator used to calculate the percentage correct also varied across sessions. 
Although this is not ideal for comparing data across sessions, it was necessary in order to assess if participants ended teaching at the correct time for each target. Since the participants were implementing DTI with actual students (and not confederates following a script), we were not able to control for the exact number of trials implemented across sessions.

In addition to measuring the overall percentage correct implementation, we also calculated the mean percentage of correct implementation for each DTI component during each condition of the study (Table 4). Social validity of the ICT was collected using a questionnaire. After completing the ICT modules, the participants completed a questionnaire regarding their experience with the ICT. The questionnaire was designed using the quiz function on Instructure Canvas and contained six questions on a Likert scale (e.g., The modules described the content clearly; I felt like there was enough information in the modules to learn how to implement DIT; I would recommend the ICT to another person interested in learning how to implement DTI) and three open-ended questions (e.g., What content did you find to be difficult to understand?; What training feature did you like the most?).

Trial-by-trial interobserver agreement (IOA) was assessed for 33\% of sessions across all conditions via videos for each participant. Interobserver agreement was calculated the same as described above, and IOA was calculated by dividing the number of agreements by the number of agreements plus disagreements and multiplying by 100 to get a percentage. An agreement was scored when both observers record the same response for each component as correct, incorrect, or not applicable. For all participants, the mean IOA was 93\% (range $85-100 \%$ ).

\section{Experimental Design and Procedures}

We used a non-concurrent multiple-baseline design across classrooms (two participant-student dyads per classroom) to evaluate the effects of the ICT on paraprofessionals' implementation of DTI with students with developmental disabilities. In order to minimize any possible carryover effects between participants, we used a multiple-baseline design across classrooms, instead of across participants. Therefore, each phase was implemented for both dyads in each classroom at the same time, such that the conditions were staggered across classrooms rather than across dyads. One to two sessions were conducted per day between 2 and 4 days per week during a 6-8-week period. If two sessions were conducted in a day, at least $30 \mathrm{~min}$ separated the two sessions.

\section{General Procedures}

Each session began with the research assistant reading a script instructing the participant to prepare the learning environment for teaching. At the same time, the research assistant gave the participant the bin containing the teaching materials and binder. For the first session, the participants were given 10 min to review the materials and set up the learning environment for teaching sessions. For all subsequent sessions, the participants were given 5 min for setup. During this time, the participant 
Table 4 Mean percent correct of DTI components across phases per participant

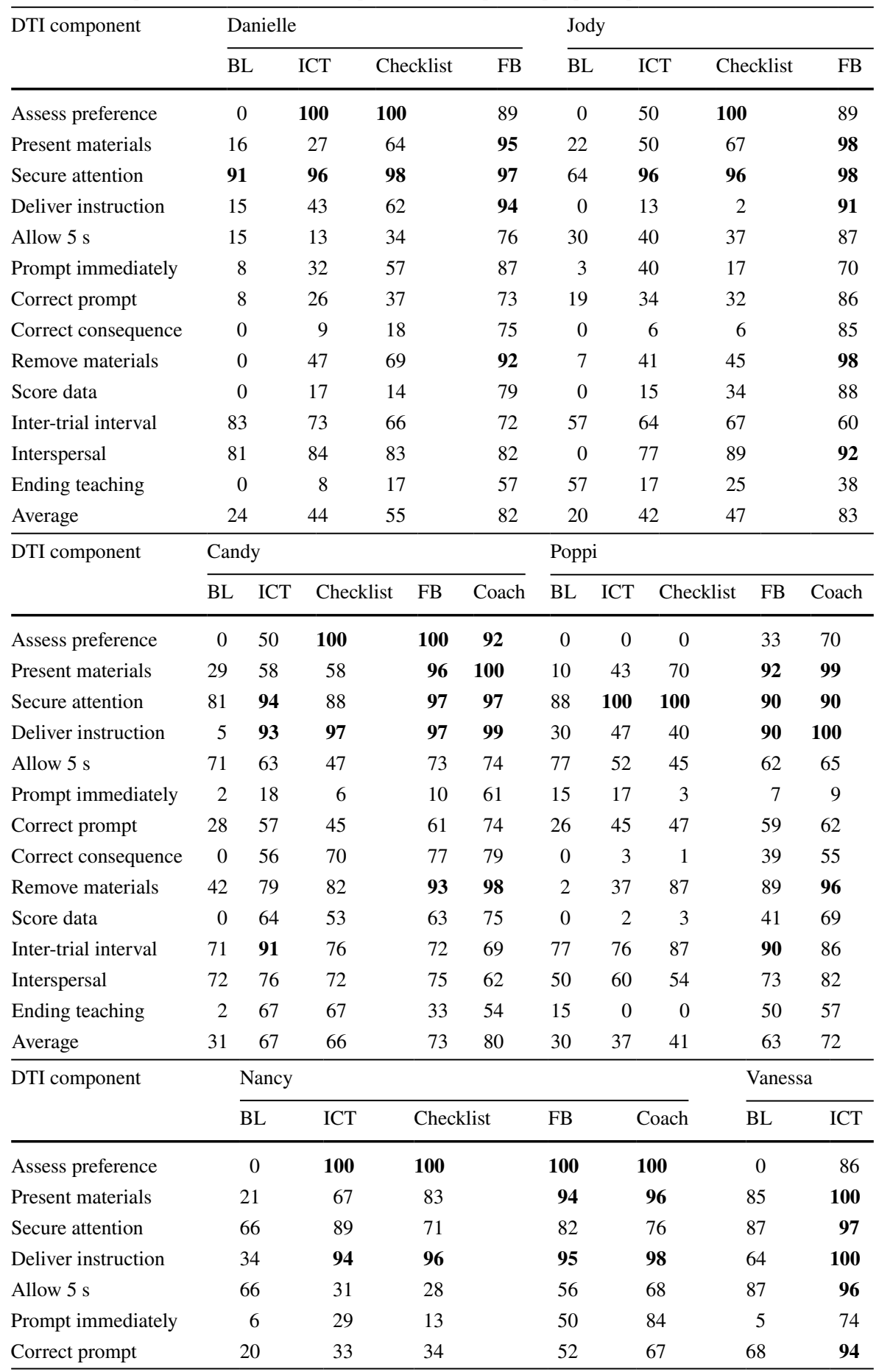


Table 4 (continued)

\begin{tabular}{|c|c|c|c|c|c|c|c|}
\hline \multirow[t]{2}{*}{ DTI component } & \multicolumn{5}{|c|}{ Nancy } & \multicolumn{2}{|c|}{ Vanessa } \\
\hline & $\mathrm{BL}$ & ICT & Checklist & FB & Coach & $\mathrm{BL}$ & ICT \\
\hline Correct consequence & 0 & 30 & 15 & 50 & 77 & 4 & 79 \\
\hline Remove materials & 6 & 44 & 67 & 88 & 93 & 46 & 98 \\
\hline Score data & 0 & 20 & 16 & 40 & 68 & 11 & 96 \\
\hline Inter-trial interval & 67 & 86 & 78 & 89 & 81 & 71 & 75 \\
\hline Interspersal & 89 & 76 & 68 & 67 & 63 & 27 & 98 \\
\hline Ending teaching & 13 & 8 & 17 & 21 & 22 & 27 & 79 \\
\hline Average & 30 & 54 & 53 & 68 & 76 & 45 & 90 \\
\hline
\end{tabular}

Components in bold indicate met performance criterion of $90 \%$ or higher accuracy

$B L$ baseline, $I C T$ interactive computerized training, $F B$ feedback

was expected to read and look over the three instructional program sheets, corresponding data sheets, and prepare materials and reinforcers for teaching.

Once the participant said she was ready, or if the time elapsed, the research assistant read another script that instructed the participant to use the student's curriculum binder for teaching and instructing her to inform the research assistant when she was finished. If the participant asked a question, the research assistant responded by saying, "I am sorry, but I cannot answer any questions at this time. Try your best and let me know when you are finished."

\section{Baseline}

Baseline sessions were conducted as described above. The materials included in the bin during baseline included materials to teach imitation, receptive identification, and expressive identification.

\section{Generalization Probe}

Generalization probes were conducted as described in the general procedures. The materials included in the bin during generalization sessions included materials to teaching receptive actions, match-to-sample, and answering simple questions.

\section{Interactive Computerized Training}

Following baseline, participants were given access to the ICT modules. Participants were instructed to complete each module in one sitting and were able to complete the module only once, in order to measure the amount of time it took for each participant to complete each module. Participants were given a deadline of 1 week, and an email reminder was provided if the deadline was not met. 


\section{Post-training}

After completing the ICT modules, participants continued implementing teaching sessions with their assigned student and sessions were conducted as described above. Sessions continued until the participant implemented the DTI components $90 \%$ correct for two consecutive sessions. If performance was below $80 \%$ following two consecutive post-ICT sessions, participants received additional training that progressed from low to more intensive feedback (see below).

\section{Teaching Checklist}

Following two sessions below $80 \%$, a teaching checklist was added to the session materials. Many errors observed during the initial post-ICT phase appeared to be related to participants not reading the instructional program and data sheets. Thus, a teaching checklist was added as a first attempt to address these errors by serving as an additional discriminative stimulus for the participants to engage in the correct sequence of behaviors. Sessions were conducted as described under the general procedures, except participants were given a one-page laminated checklist to guide their teaching session. The checklist was divided into two sections outlining the steps the participant should follow when preparing the learning environment and during teaching. The checklist included the following items: (a) read program sheets, (b) remove data sheets from binder, (c) review data sheets, (d) arrange materials and reinforcers, (e) conduct preference assessment for tangibles, (f) conduct preference assessment for edibles, (g) conduct probe trials for all teaching targets, and (h) conduct teaching trials. The participants were given $10 \mathrm{~min}$ to set up the learning environment during the first session in which the teaching checklist was implemented, and then $5 \mathrm{~min}$ for all subsequent sessions.

\section{Remote Performance Feedback}

Following two sessions below $80 \%$ with the teaching checklist, participants met with a research assistant (hereafter referred to as the coach) and received remote feedback on their performance using a similar procedure as described in LeBlanc et al. (2005). The meeting was scheduled during school hours at a convenient time for the participant to leave the classroom for 20-30 min. The coach reviewed the participant's performance during the most recent session via video and scored each component as either No (0-49\%), Some (50-89\%), or Yes (90-100\%). During the feedback session, the coach gave feedback on each DTI component. Corrective feedback was delivered for all components marked as No or Some (e.g., "Remember you should record data after every instruction; correct and incorrect responses. This is important so you can correctly prompt and respond to student errors and know when to stop teaching"). Praise was delivered for all components marked as Yes (e.g., "Great job removing materials after every teaching trial!"). The coach answered any questions; however, the coach did not model the correct performance of skills. Participants received a completed feedback form via email for their reference. The next day, participants continued sessions with their assigned student. Following every third session, the 
coach viewed their last session and delivered additional feedback. If the participant met the performance criterion, the coach delivered written feedback via email with a completed feedback form attached. If performance was below criterion, another meeting with the coach was scheduled. Participants continued receiving feedback every third session until they reached the performance criterion or if performance was still below the criterion following two remote meetings with the coach.

\section{Remote In Vivo Coaching}

If a participant did not reach the mastery criterion following two remote performance feedback meetings, she received more intensive feedback with a session of remote, in vivo coaching. The coach observed the participant's teaching session in real time through a video conference application and provided instructions, modeling, and direct feedback for $30 \mathrm{~min}$. At the end of the $30 \mathrm{~min}$, if the teaching session had not ended, the coach stopped the session to summarize the feedback delivered and answer any additional questions. The next day, participants continued implementing sessions as usual until responding stabilized using visual analysis.

\section{Maintenance}

Following the final research session, a follow-up probe was conducted at 2 weeks to assess maintenance of DTI implementation following ICT and in the absence of feedback. Maintenance was not assessed for Poppi, Candy, or Nancy because they did not reach the mastery criterion and the school year ended.

\section{Procedural Integrity}

Procedural integrity was assessed for 33\% of sessions across all conditions for each participant to ensure the research assistants implemented session procedures correctly. Data were collected per opportunity by scoring "yes" or "no" for each procedural component. The following five procedural integrity components were assessed: (a) the researcher read the correct instruction from a script to signal the participant to prepare learning environment for teaching, (b) the researcher gave the participant a bin of all the necessary materials, (c) the researcher gave the participant the allotted time to prepare the learning environment for teaching ( 5 or $10 \mathrm{~min}$ depending on the phase), (d) after the allotted time, the researcher read the correct instructions from a script to signal the participant to begin implementing DTI with her assigned student, and (e) the researcher did not provide any other feedback or instructions to the participant. Integrity data were calculated by dividing the number of "yes" by the total number of components and multiplying by 100 to yield a percentage. The mean integrity was 99\% (range 80-100\%) across all participants and conditions.

Procedural integrity was also assessed for $100 \%$ of all remote performance feedback sessions to ensure each participant received a similar experience. The same research assistant conducted all of the feedback sessions and composed all email 
Fig. 1 Percent correct of DTI components. The open shapes represent each participant's percentage of correct implementation of DTI during teaching sessions, and the closed shapes represent the generalization session. The two data paths within each panel are labeled to indicate which path represents which participant. *Indicates sessions following a remote performance feedback session with the coach. **Indicates sessions following an email with remote performance feedback

correspondences. Data were collected using a per-opportunity measure by scoring either Yes or No for each component. Then, the data were converted into a percentage by dividing the number of yes's by the total number of components and multiplying by 100. The following procedural integrity components were assessed: (a) sent an email with scheduled date and time (attachment of a blank feedback form included for initial session), (b) introduced self and oriented the participant how the meeting would proceed, (c) oriented the participant to the feedback form (initial session), (d) delivered corrective feedback for all components marked with Some or $\mathrm{No}$, (e) delivered specific praise for components marked with a Yes, (f) answered all participant's questions, (g) ended feedback by reviewing skills the participant should work on and skills to maintain, and (h) sent the participant their completed feedback form via email. For all participants, the mean integrity was 99\% (range $97-100 \%$ ).

\section{Results}

Five participants completed the module by the 1-week deadline. Candy completed the training after 8 days. It took participants an average of 305 min (range 221-353 min) to complete all six modules. Participants were able to complete modules one through six, respectively, in an average of $33 \mathrm{~min}$ (range 25-40 min), 45 min (range 23-70 min), 47 min (range 32-82 min), 55 min (range 24-74 min), $64 \mathrm{~min}$ (range 51-85 min), and $61 \mathrm{~min}$ (range 24-102 min).

Figure 1 depicts the percentage of DTI components implemented correctly for each participant. The open shapes represent sessions in which the participants were implementing teaching sessions with the target programs taught in the ICT modules (non-vocal imitation, receptive identification, and expressive identification). The closed shapes represent generalization sessions in which the participants implemented teaching with the generalization programs that were not taught in the ICT modules (receptive actions, match-to-sample, and answering simple questions). Each data path represents a different participant-student dyad within each classroom (labeled on the figure). Table 4 shows the percentage of correct implementation of DTI components across the each condition. Common DTI integrity errors across all participants included: (a) implementing the errorless learning procedure, (b) delivering correct consequence, (c) inter-trial interval, (d) scoring data correctly, and (e) ending teaching of targets.

Danielle and Jody's data are presented in the upper panel of Fig. 1. During baseline, both Danielle and Jody demonstrated low integrity of the DTI components, an average of $28 \%$ and $20 \%$, respectively. Their performance during the baseline generalization probe was also around $25 \%$. Following the completion of the ICT, the percentage of correct implementation of DTI increased for both participants to about 


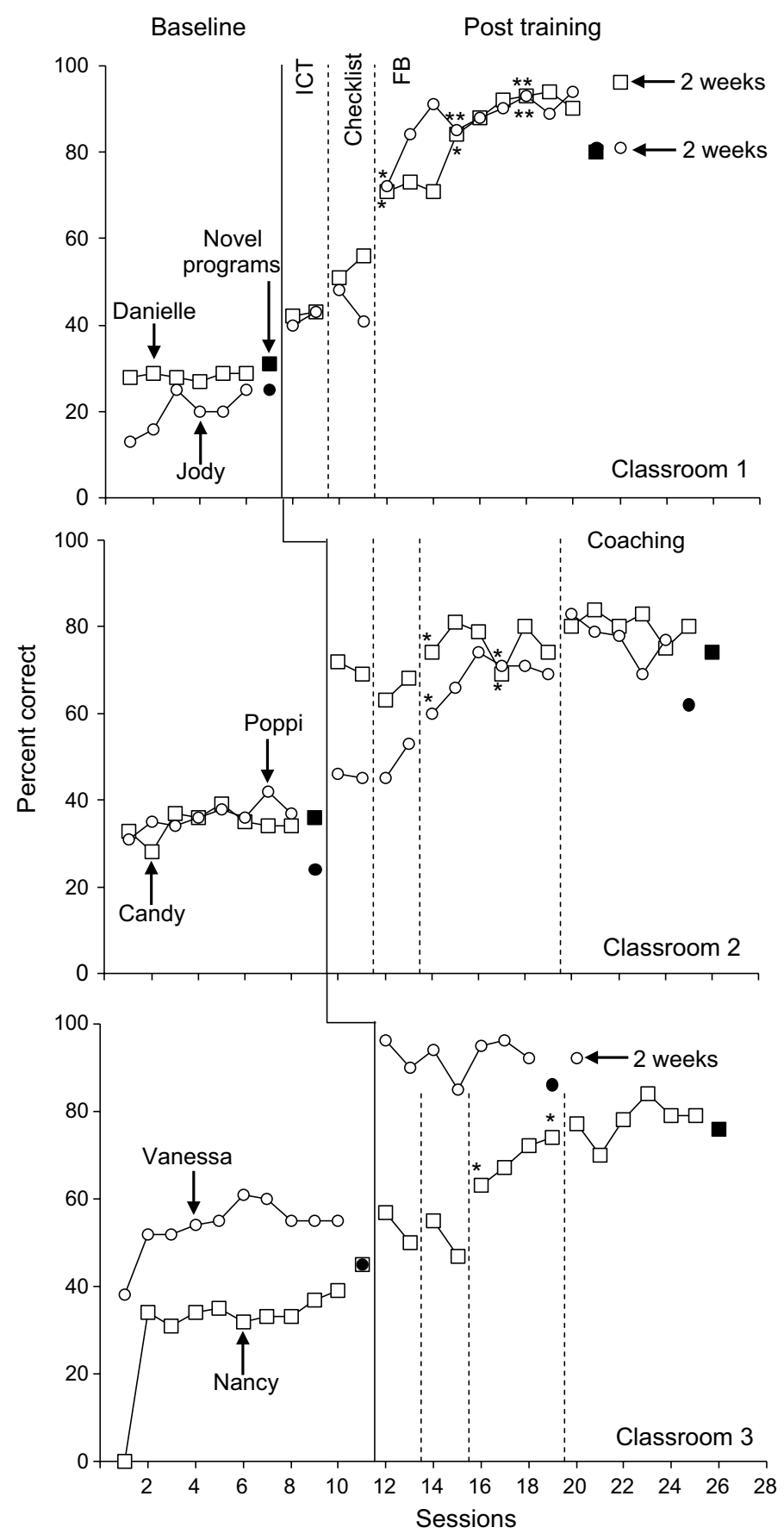


43\%. Because both Danielle's and Jody's performance was below $80 \%$ for two sessions following the ICT training, we implemented the teaching checklist. Following two sessions with the checklist, Danielle's performance increased slightly to $54 \%$, and Jody's performance remained the same around 40\%. Both Danielle and Jody received the remote performance feedback between sessions 11 and 12 (indicated by the $*$ on the graph), and we observed an increase in performance for both at session 12 to about $70 \%$. Jody's performance continued to increase until her implementation of DTI reached the mastery criterion $(90 \%)$ at session 14, which she maintained for the rest of the study. Following the remote performance feedback, Danielle's performance remained around $70 \%$ for three sessions. Another remote performance feedback session was conducted between sessions 14 and 15, at which point her performance increased to $82 \%$. Danielle's implementation of DTI continued to increase until she met the mastery criteria at session 17 , and her percentage of correct implementation remained high for the rest of the study. Refer Table 4 to see the DTI components that each participant completed with a high percentage of accurate implementation across each phase. Both Jody and Danielle continued to receive email feedback (indicated by the $*^{*}$ on the graphs) once they met the mastery criterion. Danielle and Jody's performance remained high during a generalization probe to three untrained instructional programs, respectively, at $80 \%$ and $81 \%$, and remained high during the 2-week follow-up check, respectively, at $96 \%$ and $81 \%$ integrity.

Candy and Poppi's data are presented in the middle panel of Fig. 1. The accuracy of their implementation of DTI components was low and stable during baseline around $35 \%$ and $36 \%$. Following the completion of the ICT, accuracy of implementation of DTI components increased to $71 \%$ for Candy and $46 \%$ for Poppi. Because both participants' performance was below $80 \%$ for two sessions, the teaching checklist was introduced after session 11. Following the introduction of the teaching checklist, Candy's performance dropped slightly to 66\% (although an increase in accuracy in other components was observed, see Table 3) and Poppi's performance increased slightly to $49 \%$. Prior to session 14 , both participants received the remote performance feedback. Candy's integrity of implementation of DTI increased to 78\% and plateaued, and Poppi's performance gradually increased after feedback to around $70 \%$ following two sessions with the coach. Because both Candy's and Poppi's performance was still below $80 \%$ following two sessions with remote feedback, we implemented remote in vivo coaching following session 19. Participants' integrity of implementation of DTI components stabilized around $80 \%$ after remote in vivo feedback. During the generalization probe, Candy's integrity of implementation of DTI components remained around the same integrity level, 74\%. Candy's and Poppi's performance increased during a generalization probe compared to baseline to three untrained instructional programs, respectively, at $74 \%$ and $62 \%$,

Vanessa and Nancy's data are shown in the bottom panel of Fig. 1. Vanessa had the highest baseline and averaged 54\% accurate implementation of DTI components. Nancy's accuracy of DTI components was low during baseline around 30\%. During the first baseline session, Nancy did not conduct any target instructions and played with the student for several minutes and then said she was done. Following ICT, Vanessa's accuracy of implementation of DTI components immediately increased to criterion and stabilized around 93\%. Her integrity of DTI dropped in session 15 
because she failed to conduct the preference assessments. Nancy's post-ICT performance increased to $54 \%$. Nancy followed the same additional training components as the previous participants. Following the introduction of the teaching checklists, her performance did not change (51\%). Remote performance feedback was provided prior to session 16. Nancy's implementation of DTI components increased and gradually increased from $63 \%$, to $67 \%$, to $73 \%$. Nancy frequently made errors related to the errorless learning procedure and the delivery of appropriate consequences (Table 4). Nancy received another feedback session with the coach before session 19. Only a $2 \%$ increase was observed in the next teaching session, thus indicating a need for more extensive feedback. Remote in vivo coaching was introduced prior to session 20. Following remote in vivo coaching, her integrity of implementation of DTI components only increased slightly, but her integrity implementing the errorless learning procedure increased from an average of 52 to $70 \%$ (Table 4). Her performance stabilized below the performance criterion at $78 \%$ proficiency. During the generalization probe to novel programs, Vanessa's integrity of implementation of the DTI components remained high at $86 \%$ and Nancy's performance was relatively similar to treatment sessions at $76 \%$. Vanessa's performance remained high during the 2 -week follow-up check at $92 \%$.

\section{Social Validity Questionnaire}

After the completion of the training modules, participants completed a questionnaire regarding their experience (Table 5). Overall, participants rated the six Likert questions with either agree or strongly agree. All participants rated the modules as informative and would recommend the training other interested in learning DTI with either agree or strongly agree. A neutral was marked for three questions regarding interest, clarity, and amount of content. All participants reported they liked the videos and interactive activities embedded in the modules. Three participants reported data collection, Module 6, had the most difficult instructional content.

\section{Discussion}

The present study found that ICT alone and ICT plus additional remote training components can increase paraprofessional's implementation DTI procedures for teaching individuals with developmental disabilities, although the effectiveness of these procedures varied across paraprofessional participants. One participant met criterion (i.e., $90 \%$ or higher fidelity) following the ICT alone. Two additional participants met criterion following ICT and remote feedback, and three participants reached around $80 \%$ proficiency following ICT, remote feedback, and remote in vivo coaching. All participants' accuracy of implementation of DTI increased during the generalization probe, indicating that participants were able to generalize the skills to three novel instructional programs. Participants who met the performance criterion maintained high levels of integrity at a 2 -week follow-up probe. Although the results of this study were mixed, in that the participants required varying levels of support 


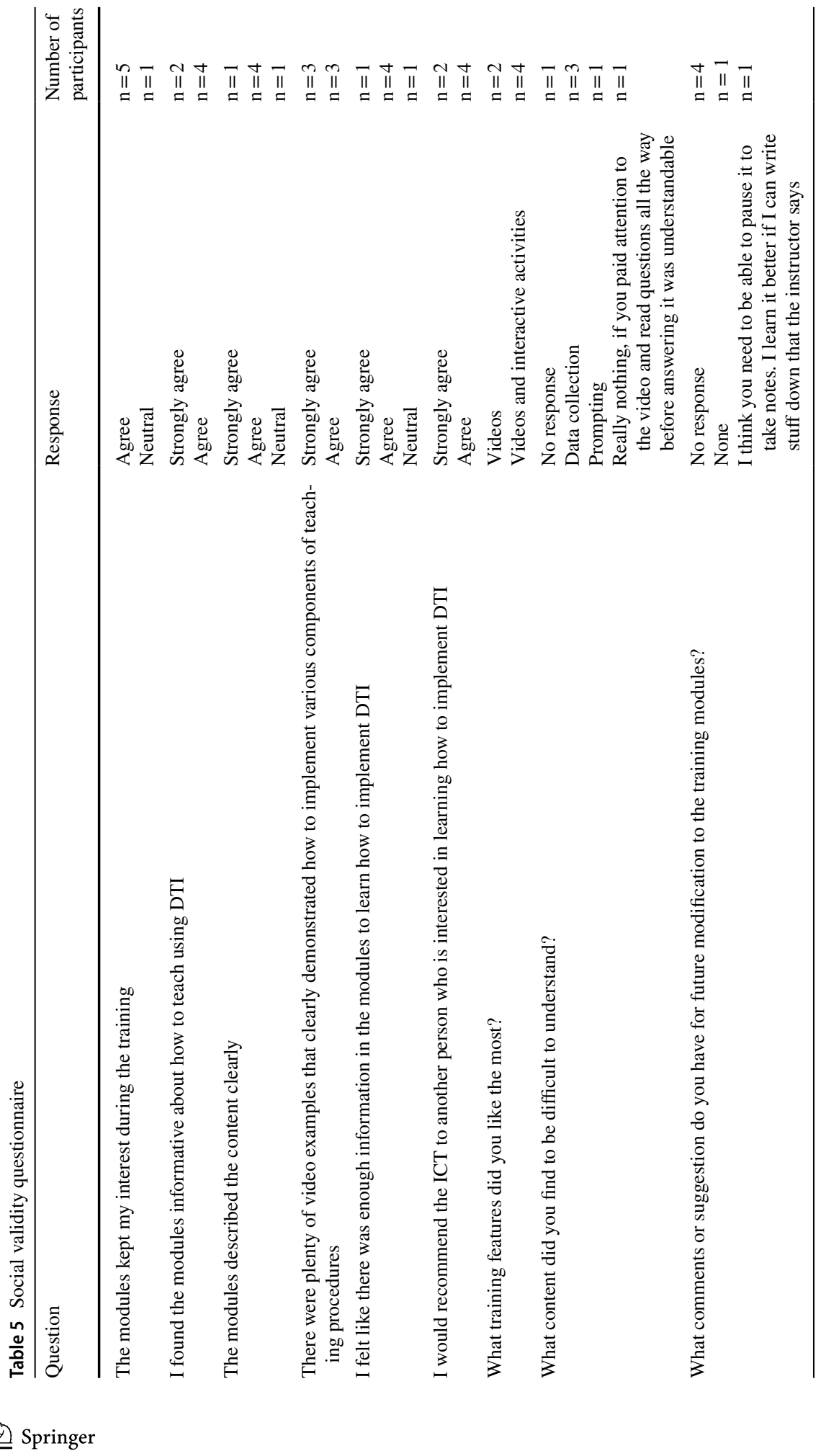


to increase their teaching integrity to $80 \%$ or higher, it only took $0-150 \mathrm{~min}$ of a professional's time to provide additional feedback and coaching. Each extension and implications for future research will be discussed below.

The first extension of this study was the use of ICT to teach paraprofessionals to implement DTI. An immediate increase in the accuracy of implementation of DTI components following ICT was observed for two participants, Candy and Vanessa. The accuracy of other participants increased slightly following the ICT, and additional training components were required to produce greater changes. There are several reasons why participants from this study may have not performed as well as in the previous studies (Higbee et al. 2016; Pollard et al. 2014), such as different participant characteristics and learning histories as well as increased complexity of the DTI skills taught and evaluated. However, it is important to note that in the previous studies (Higbee et al. 2016; Pollard et al. 2014) several participants also required additional feedback in order to reach the performance criterion of $85 \%$ with a student with ASD. Given the complexity of DTI and potential differences in participant's characteristics, it may not be surprising that some participants required additional training components. In addition, these results align with previous implementation research by Joyce and Showers (2002), in that learning a new skill that involves a more complex repertoire (e.g., implementing an errorless learning procedure in addition to DTI) requires additional training components such as feedback and coaching in order to obtain transfer of the skill into practice.

A second extension, expanded on the complexity of DTI skills taught within ICT to included prompts and prompting fading which is an integral part of DTI. Previous ICT studies (Higbee et al. 2016; Pollard et al. 2014) only assessed the participants' ability to provide a prompt and increase prompts following consecutive errors across a small number of learning trials (15-20). However, they did not assess participants' ability to fade prompts. In the current study, learning trials were increased to evaluate the errorless learning procedural components (i.e., probe trials, fading prompts, error correction) and more closely mimic a typical one-on-one teaching session. Post-training teaching sessions were approximately $30 \mathrm{~min}$ long, which included conducting preference assessments, between 6 and 12 probe trials, and between 30 and 60 teaching trials interspersing instructional targets within and across three programs. All participants, except Vanessa, had low procedural integrity when implementing the errorless learning procedures. The results of this study are similar to findings of those in Cook et al. (2015) and Brand et al. (2017) who observed integrity errors during the error correction procedure for novice and experienced instructors. Unfortunately, inaccurate implementation of prompting and error correction procedures may negatively impact student learning (e.g., prompt dependency, increase errors, delay acquisition). Perhaps, the addition of the errorless learning procedure increased the difficulty level for paraprofessionals to implement DTI with accuracy following the ICT alone.

In addition to the errorless learning procedure, we taught participants to conduct a brief MSWO preference assessment and to deliver the top ranked items paired with varied praise for correct responses. If a participant did not conduct the preference assessments before starting the session, then reinforcement delivery was automatically scored as incorrect for every learning trial even if praise was varied. Because 
of this strict requirement, many participants lost points on the fidelity checklist. This error was particularly detrimental to Vanessa when a decrease in integrity was observed during session 15 and Poppi because she failed to accurately conduct the assessment until she received extended feedback and coaching. Failing to provide any reinforcement is likely to have a very different effect that failing to give varied praise; it may be valuable for future studies to report these separately as opposed to within one measure on the appropriate delivery of a consequence. Researchers may want to define some of these fidelity components more loosely or parse out the components further to provide a more accurate representation of integrity and to identify the critical components that affect student's rate of acquisition. Furthermore, a continuous reinforcement schedule was used in this study for ease of data collection on integrity; however, it typically only recommended for establishing new skills.

Although these modules were developed and modified from existing ICT studies and self-instructional manual training studies, these particular modules were untested prior to the study. It is possible that the modules used in the current study would also have been insufficient to teach college students and special education teachers to implement these more advanced DTI components. It is also possible, however, that paraprofessionals may have not have responded as well to the training compared to college students and special education teachers due to potential learning histories and motivational variables. For example, all participants had an extensive working history as a paraprofessional and received no formal training to work with students with developmental disabilities. The majority of their training was informal, "on-the-job" feedback from their classroom teacher when she had time to provide it. Thus, it is possible that participants developed a learning history of teaching using certain procedures that they had implemented over several years. Because of this learning history, it is possible that it competed with learning a new way to teach similar skills. As an example, Poppi often failed to end the teaching trial following an incorrect response. Instead, when the student responded incorrectly, she would immediately prompt the correct response and deliver reinforcement. This is problematic, because the student may learn to chain the two responses together. Because this response was at strength in Poppi's repertoire prior to the study, it may have interfered with the implementation of a new teaching procedure.

Another common error made across all participants was their insufficient use of time during the setup time to read over the provided materials (i.e., instructional program sheet, data sheets, teaching checklist). Instructional program sheets were provided for all six skills taught, which provided details on the instruction, materials, a brief overview on how to teach the skill, a definition of the correct response, prompt hierarchy, prompt fading and error correction rules, and data collection. It is likely that many participants made several errors due to their failure to read and refer to the program sheets and data sheets. For example, many of the participants incorrectly ran the expressive identification program as a receptive identification program. Participants also failed to deliver the correct instruction and failed to present the material correctly on numerous occasions. Similar errors were made during the generalization probe with three untrained programs. These errors could have been caused by unfamiliarity with structured teaching programs, poor layout or organization of the instructional program sheets, or even reading comprehension challenges 
of participants. Although teaching checklist was provided to help with this problem, future researchers may want to investigate these factors in future studies.

In previous studies, performance feedback and coaching was delivered in person. Thus, a third extension was to evaluate providing feedback and coaching from a distance. The results of this study demonstrate that feedback and coaching can be delivered from a distance_-although the results of participant integrity scorings were variable. Following two sessions of remote performance feedback, Danielle reached the performance criterion of $90 \%$ accuracy. Jody only required one feedback session, and check-in feedback was delivered via email. Candy and Poppi's integrity increased following remote performance feedback sessions, but performance was still below criterion. Thus, a session of remote in vivo coaching was provided and they both reached about $80 \%$ proficiency. Although all participants increased their integrity of implementation of DTI components, some participants, such as Nancy, may require more intensive training methods (e.g., motivational or consequence-based interventions) to accept and implement feedback to reach proficient levels. In addition, some DTI components may require more feedback (e.g., prompting and error correction procedures), but ICT can teach some of the foundational skills to mastery, such as gaining the student's attention and correct presentation of discriminative stimuli. Although data were not collected on efficiency to compare the resources of remote feedback compared to in person feedback, remote feedback can be used as a supplemental training tool for those that need more support to reach proficiency and only took the coach $0-150 \mathrm{~min}$. It is likely remote feedback and ICT would be more cost-effective compared to traditional in person BST, if the training volume is high, such as school districts, according to Geiger et al. (2018) estimate of return on investment.

In summary, school districts tend to rely heavily on traditional face-to-face training methods and the classroom teachers to train paraprofessionals, which may possibly have little impact on the performance of paraprofessionals. One benefit of ICT is that trainees can access the training on their own time and complete it at their own pace. ICT can incorporate the BST training components of instruction, modeling, and feedback into an engaging training package. From previous research studies, ICT appeared to be an effective teaching tool. Due to the increase in complexity of DTI skills and new population, it is unknown which variable or combination of variables contributed to lower levels of participant performance found in the current study and future research is necessary. However, performance feedback and coaching delivered from a distance was successful in increasing procedural integrity and has been documented as a critical component for transfer of skills into practice (Joyce and Showers 2002). Because several remote feedback checks were needed for some participants, future researchers may want to investigate the effects of training a behavioral specialists or the classroom teacher to deliver the additional performance feedback and coaching components. ICT can provide participants with foundational knowledge, but some accountability and feedback will likely also be needed. ICT may still be a potential solution to the current challenges school districts face with training paraprofessionals on effective teaching strategies. Additional research in this area is needed to learn more about the boundaries of using ICT in order to serve the populations these training methods are intended. In addition, more research, 
such as a component analysis, is needed to identify the critical components of the ICT package to continue to develop cost-effective trainings to train at a larger scale.

Acknowledgements We thank Tim Slocum, Tyra Sellers, Robert Morgan, and Eadric Bressel for their comments on an earlier draft of this manuscript. We also thank Michele, Neeley, Laura, and the school district for their valuable support throughout this project. Portions of this article were submitted in partial fulfillment of the first author's dissertation under the direction of the second author.

Funding This study was partially fund by a grant from Utah State University, Graduate Research and Collaborative Opportunities Grant.

\section{Compliance with Ethical Standards}

Conflict of interest The authors declare that they have no conflict of interest.

Ethical Approval All procedures performed in studies involving human participants were in accordance with the ethical standards of the institutional and/or national research committee and with the 1964 Declaration of Helsinki and its later amendments or comparable ethical standards.

Informed Consent Informed consent was obtained from all individual participants included in the study.

\section{References}

Boomer, L. W. (1994). The utilization of paraprofessionals in programs for students with autism. Focus on Autism and other Developmental Disabilities, 9, 1-9. https://doi.org/10.1177/108835769400900 201.

Brand, D., Elliffe, D., \& DiGennaro Reed, F. D. (2017). Using sequential analysis to assess component integrity of discrete-trial teaching programs. European Journal of Behavior Analysis, 19, 1-18. https://doi.org/10.1080/15021149.2017.1404392.

Carr, J. E., Nicolson, A. C., \& Higbee, T. S. (2000). Evaluation of a brief multiple-stimulus preference assessment in a naturalistic context. Journal of Applied Behaviors Analysis, 33, 353-357. https:// doi.org/10.1901/jaba.2000.33-353

Cook, J. E., Subramaniam, S., Brunson, L. Y., Larson, N. A., Poe, S. G., \& St. Peter, C. C. (2015). Global measures of treatment integrity may mask important errors in discrete-trial training. Behavior Analysis in Practice, 8, 37-47. https://doi.org/10.1007/s40617-014-0039-7.

Fazzio, D., \& Martin, G. L. (2011). Discrete-trials teaching with children with autism: A self-instructional manual. Winnipeg: Hugo Science Press.

Fixsen, D. L., Naoom, S. F., Blase, K. A., Friedman, R. M. \& Wallace, F. (2005). Implementation research: A synthesis of the literature. Tampa, FL: University of South Florida, Louis de la Parte Florida Mental Health Institute, The National Implementation Research Network (FMHI Publication \#231).

Geiger, K. B., LeBlanc, L. A., Hubik, K., Jenkins, S. R., \& Carr, J. E. (2018). Live training versus e-learning to teach implementation of listen response programs. Journal of Applied Behavior Analysis, 51, 220-235. https://doi.org/10.1002/jaba.444.

Higbee, T. S., Aporta, A. P., Resende, A., Nogueira, M., Goyos, C., \& Pollard, J. S. (2016). Interactive computer training to teach discrete trial instruction to undergraduates and special educators in Brazil. Journal of Applied Behavior Analysis, 49, 780-793. https://doi.org/10.1002/jaba.329.

Joyce, B., \& Showers, B. (2002). Student Achievement Through Staff Development (3rd ed.). Alexandria, VA: Association for Supervision and Curriculum Development.

Leblanc, M. P., Ricciardi, J. N., \& Luiselli, J. K. (2005). Improving discrete trial instruction by paraprofessional staff through abbreviated performance feedback intervention. Education and Treatment of Children, 28, 76-82. 
Pollard, J. S., Higbee, T. S., Akers, J. S., \& Brodhead, M. T. (2014). An evaluation of interactive computer training to teach instructors to implement discrete trials with children with autism. Journal of Applied Behavior Analysis, 47, 765-776. https://doi.org/10.1002/jaba.152.

Riggs, C. G., \& Mueller, P. H. (2001). Employment and utilization of paraeducators in inclusive settings. The Journal of Special Education, 35, 54-62. https://doi.org/10.1177/002246690103500106.

Sarokoff, R. A., \& Sturmey, P. (2004). The effects of behavioral skills training on staff implementation of discrete-trial teaching. Journal of Applied Behavior Analysis, 37, 535-538. https://doi.org/10.1901/ jaba.2004.37-535.

Severtson, J. M., \& Carr, J. E. (2012). Training novice instructors to implement errorless discrete-trial teaching: A sequential analysis. Behavior Analysis in Practice, 5, 13-23.

U.S. Department of Education. (2014). 36th annual report to congress on the implementation of the individuals with disabilities education act. Retrieved from http://www.ed.gov/about/reports/annual/ osep. 